\title{
Second-Order Differential Equation: Oscillation Theorems and Applications
}

\author{
Shyam S. Santra, ${ }^{1}$ Omar Bazighifan $\mathbb{D}^{2,3}$ Hijaz Ahmad ${ }^{1},{ }^{4,5}$ and Yu-Ming Chu $\mathbb{D}^{6,7}$ \\ ${ }^{1}$ Department of Mathematics, JIS College of Engineering, Kalyani 741235, India \\ ${ }^{2}$ Department of Mathematics, Faculty of Science, Hadhramout University, Hadhramout, Yemen \\ ${ }^{3}$ Department of Mathematics, Faculty of Education, Seiyun University, Hadhramout, Yemen \\ ${ }^{4}$ Department of Basic Sciences, University of Engineering and Technology, Peshawar, Pakistan \\ ${ }^{5}$ Section of Mathematics, International Telematic University Uninettuno, Corso Vittorio Emanuele II, 39, Roma 00186, Italy \\ ${ }^{6}$ Department of Mathematics, Huzhou University, Huzhou 313000, China \\ ${ }^{7}$ Hunan Provincial Key Laboratory of Mathematical Modeling and Analysis in Engineering, Changasha, \\ University of Science \& Technology, Changsha 410114, China
}

Correspondence should be addressed to Yu-Ming Chu; chuyuming@zjhu.edu.cn

Received 12 September 2020; Revised 8 October 2020; Accepted 12 October 2020; Published 31 October 2020

Academic Editor: Fateh Mebarek-Oudina

Copyright (C) 2020 Shyam S. Santra et al. This is an open access article distributed under the Creative Commons Attribution License, which permits unrestricted use, distribution, and reproduction in any medium, provided the original work is properly cited.

\begin{abstract}
Differential equations of second order appear in a wide variety of applications in physics, mathematics, and engineering. In this paper, necessary and sufficient conditions are established for oscillations of solutions to second-order half-linear delay differential equations of the form $\left(\varsigma(y)\left(u^{\prime}(y)\right)^{a}\right)^{\prime}+p(y) u^{c}(\vartheta(y))=0$, for $y \geq y_{0}$, under the assumption $\int^{\infty}(\varsigma(\eta))^{-(1 / a)}=\infty$. Two cases are considered for $a<c$ and $a>c$, where $a$ and $c$ are the quotients of two positive odd integers. Two examples are given to show the effectiveness and applicability of the result.
\end{abstract}

\section{Introduction}

Differential equations (DEs) have received a lot of attention, and it is an active research area among scientists and engineers [1-8]. The DEs have ability to formulate many complex phenomena in various fields such as biology, fluid mechanics, plasma physics, fluid mechanics, and optics; many exact and numerical schemes have been being derived such as [9-15]. Differential equation of second order appears in models as well as in physical applications such as fluid dynamics, electromagnetism, acoustic vibrations and quantum mechanics, biological, physical and chemical phenomena, optimization, mathematics of networks, and dynamical systems (see [16-24]).

In this article, we consider the differential equation

$$
\left(\varsigma(y)\left(u^{\prime}(y)\right)^{a}\right)^{\prime}+p(y) u^{c}(\vartheta(y))=0, \quad \text { for } y \geq y_{0},
$$

where $a$ and $c$ are the quotient of two positive odd integers, and the functions $p, \varsigma$, and $\vartheta$ are continuous that satisfy the conditions stated below:

(A1) $\vartheta \in C([0, \infty), \mathbb{R}), \vartheta(y)<y, \lim _{y \rightarrow \infty} \vartheta(y)=\infty$.

(A2) $\varsigma \in C^{1}([0, \infty), \mathbb{R}), \quad p \in C([0, \infty), \mathbb{R}) ; 0<\varsigma(y)$, $0 \leq p(y)$ for all $y \geq 0 ; p(y)$ is not identically zero in any interval $[b, \infty)$.

(A3) $\Upsilon(y)=\int_{y_{1}}^{y} \varsigma^{-1 / a}(\eta) \mathrm{d} \eta$ with $\lim _{y \longrightarrow \infty} \Upsilon(y)=\infty$. (A4) the existence of a differentiable function $\vartheta_{0}$ such that $0<\vartheta_{0}(y) \leq \vartheta(y)$, for $\vartheta_{0}^{\prime}(y) \geq \vartheta_{0}>0$, for $y \geq y_{0}$.

In $[25,26]$, Baculǐkovă and Džurina have considered

$$
\begin{gathered}
\left(\varsigma(y)\left(z^{\prime}(y)\right)^{a}\right)^{\prime}+p(y) u^{c}(\vartheta(y))=0, \\
z(y)=u(y)+q(y) u(\tau(y)), \quad y \geq y_{0},
\end{gathered}
$$


and obtained oscillation criteria for the solutions of (2) using comparison techniques when $a=c=1,0 \leq q(y)<\infty$, and $\lim _{y \rightarrow \infty} \Upsilon(y)=\infty$. In the same technique, Džurina and Dzurina [27] have studied the oscillatory behavior of the solutions of (2) under the assumptions $0 \leq q(y)<\infty$ and $\lim _{y \rightarrow \infty} \Upsilon(y)=\infty$. In [28], Bohner et al. have studied the oscillatory behavior of solutions of (2) under $a=c$, $\lim _{y \rightarrow \infty} \Upsilon(y)<\infty$, and $0 \leq q(y)<1$. Grace et al. [29] have studied the oscillatory behavior of (2) when $a=c$ and $\lim _{y \rightarrow \infty} \Upsilon(y)<\infty$ and $\quad \lim _{y \rightarrow \infty} \Upsilon(y)=\infty$ and $0 \leq q(y)<1$. In [30], Ali has studied the oscillatory behavior of the solutions of (2), under the assumptions $\lim _{y \rightarrow \infty} \Upsilon(y)<\infty$ and $q(y) \geq 0$. Karpuz and Santra [31] have studied the oscillatory behavior of

$$
\left(\varsigma(y)(u(y)+q(y) u(\tau(y)))^{\prime}\right)^{\prime}+p(y) f(u(\vartheta(y)))=0,
$$

by considering the assumptions $\lim _{y \longrightarrow \infty} \Upsilon(y)<\infty$ and $\lim _{y \rightarrow \infty} \Upsilon(y)=\infty$ for different ranges of the neutral coefficient $q$.

For further work on the oscillation of this type of equations, we refer the readers to the references. Note that the majority of works consider only sufficient conditions, and merely a few consider both necessary and sufficient conditions. Hence, the objective of this work is to establish both necessary and sufficient conditions for the oscillation of solutions of (1) without using the comparison and the Riccati techniques. In this paper, we restrict our attention to the study (1), which includes the class of functional differential equations of neutral type.

Remark 1. When the domain is not specified explicitly, all functional inequalities considered in this paper are assumed to hold eventually, i.e., they are satisfied for all $y$ large enough.

\section{Necessary and Sufficient Conditions}

Lemma 1. Let (A1)-(A3) hold and that $u$ is an eventually positive solution of (1). Then, there exist $y_{1} \geq y_{0}$ and $d>0$ such that

$$
\begin{array}{r}
0<u(y) \leq d \Upsilon(y), \\
\Upsilon(y)\left[\int_{y}^{\infty} p(\zeta) u^{c}(\vartheta(\zeta)) \mathrm{d} \zeta\right]^{1 / a} \leq u(y),
\end{array}
$$

for $y \geq y_{1}$.

Proof. Let $u$ be an eventually positive solution of (1). Then, by (A1), there exists a $y^{*}$ such that $u(y)>0$ and $u(\vartheta(y))>0$ for all $y \geq y^{*}$. From (1) it follows that

$$
\left(\varsigma(y)\left(u^{\prime}(y)\right)^{a}\right)^{\prime}=-p(y) u^{c}(\vartheta(y)) \leq 0 .
$$

Therefore, $\varsigma(y)\left(u^{\prime}(y)\right)^{a}$ is nonincreasing for $y \geq y^{*}$. Next, we show that $\varsigma(y)\left(u^{\prime}(y)\right)^{a}$ is positive. By contradiction, assume that $\varsigma(y)\left(u^{\prime}(y)\right)^{a} \leq 0$ at a certain time $y \geq y^{*}$. Using that $p$ is not identically zero on any interval $[b, \infty)$ and by $(6)$, there exists $y_{1} \geq y^{*}$ such that

$$
\varsigma(y)\left(u^{\prime}(y)\right)^{a} \leq \varsigma\left(y_{1}\right)\left(u^{\prime}\left(y_{1}\right)\right)^{a}<0, \quad \text { for all } y \geq y_{1} .
$$

Recall that $a$ is the quotient of two positive odd integers. Then,

$$
u^{\prime}(y) \leq\left(\frac{\varsigma\left(y_{1}\right)}{\varsigma(y)}\right)^{1 / a} u^{\prime}\left(y_{1}\right), \quad \text { for } y \geq y_{1} .
$$

Integrating from $y_{2}$ to $y$, we have

$$
u(y) \leq u\left(y_{1}\right)+\left(\varsigma\left(y_{1}\right)\right)^{1 / a} u^{\prime}\left(y_{1}\right) \Upsilon(y) .
$$

By (A3), the right-hand side approaches $-\infty$; then, $\lim _{y \rightarrow \infty} u(y)=-\infty$. This is a contradiction to the fact that $u(y)>0$. Therefore, $\varsigma(y)\left(u^{\prime}(y)\right)^{a}>0$ for all $y \geq y^{*}$. From $\varsigma(y)\left(u^{\prime}(y)\right)^{a}$ being nonincreasing, we have

$$
u^{\prime}(y) \leq\left(\frac{\varsigma\left(y_{1}\right)}{\varsigma(y)}\right)^{1 / a} u^{\prime}\left(y_{1}\right), \quad \text { for } y \geq y_{1} .
$$

Integrating this inequality from $y_{1}$ to $y$ and using that $u$ is continuous,

$$
u(y) \leq u\left(y_{1}\right)+\left(\varsigma\left(y_{1}\right)\right)^{1 / a} u^{\prime}\left(y_{1}\right) \Upsilon(y) .
$$

Since $\lim _{y \rightarrow \infty} \Upsilon(y)=\infty$, there exists a positive constant $d$ such that (4) holds.

Since $\varsigma(y)\left(u^{\prime}(y)\right)^{a}$ is positive and nonincreasing, $\lim _{y \rightarrow \infty} \varsigma(y)\left(u^{\prime}(y)\right)^{a}$ exists and is nonnegative. Integrating (1) from $y$ to $b$, we have

$$
\varsigma(b)\left(u^{\prime}(b)\right)^{a}-\varsigma(y)\left(u^{\prime}(y)\right)^{a}+\int_{y}^{\infty} p(\eta) u^{c}(\vartheta(\eta)) \mathrm{d} \eta=0 .
$$

Letting limit as $b \longrightarrow \infty$, we get

$$
\varsigma(y)\left(u^{\prime}(y)\right)^{a} \geq \int_{y}^{\infty} p(\eta) u^{c}(\vartheta(\eta)) \mathrm{d} \eta
$$

Then,

$$
u^{\prime}(y) \geq\left[\frac{1}{\varsigma(y)} \int_{y}^{\infty} p(\eta) u^{c}(\vartheta(\eta)) \mathrm{d} \eta\right]^{1 / a} .
$$

Since $u\left(y_{1}\right)>0$, integrating the above inequality yields

$$
u(y) \geq \int_{y_{1}}^{y}\left[\frac{1}{\varsigma(\eta)} \int_{\eta}^{\infty} p(\zeta) u^{c}(\vartheta(\zeta)) \mathrm{d} \zeta\right]^{1 / a} \mathrm{~d} \eta
$$

Since the integrand is positive, we can increase the lower limit of integration from $\eta$ to $y$ and then use the definition of $\Upsilon(y)$ to obtain

$$
u(y) \geq \Upsilon(y)\left[\int_{y}^{\infty} p(\zeta) u^{c}(\vartheta(\zeta)) \mathrm{d} \zeta\right]^{1 / a},
$$

which yields (5).

Theorem 1. Assume that there exists a constant $b_{1}$, the quotient of two positive odd integers, such that $0<c<b_{1}<a$. 
If (A1)-(A3) hold, then each solution of (1) is oscillatory if and only if

$$
\int_{0}^{\infty} p(\zeta) \Upsilon^{c}(\vartheta(\zeta)) \mathrm{d} \zeta=\infty
$$

Proof. On the contrary, we assume that $u$ is eventually positive solution. So, Lemma 1 holds, and then there exists $y_{1} \geq y_{0}$ such that

$$
u(y) \geq \Upsilon(y) w^{1 / a}(y) \geq 0, \quad \text { for } y \geq y_{1},
$$

where

$$
w(y)=\int_{y}^{\infty} p(\zeta) u^{c}(\vartheta(\zeta)) \mathrm{d} \zeta
$$

Computing the derivative of $w$, we have

$$
w^{\prime}(y)=-p(y) u^{c}(\vartheta(y)) .
$$

Thus, $w$ is nonnegative and nonincreasing. Since $u>0$, by (A2), it follows that $p(y) u^{c}(\vartheta(y))$ cannot be identically zero in any interval $[b, \infty)$; thus, $w^{\prime}$ cannot be identically zero, and $w$ cannot be constant on any interval $[b, \infty)$. Therefore, $w(y)>0$ for $y \geq y_{1}$. Computing the derivative, we have

$$
\left(w^{1-b_{1} / a}(y)\right)^{\prime}=\left(1-\frac{b_{1}}{a}\right) w^{-b_{1} / a}(y) w^{\prime}(y) .
$$
have

Integrating (21) from $y_{2}$ to $y$ and using that $w>0$, we

$$
\begin{aligned}
w^{1-b_{1} / a}\left(y_{2}\right) & \geq\left(1-\frac{b_{1}}{a}\right)\left[-\int_{y_{2}}^{y} w^{-b_{1} / a}(\zeta) w^{\prime}(\zeta) \mathrm{d} \zeta\right] \\
& =\left(1-\frac{b_{1}}{a}\right)\left[\int_{y_{2}}^{y} w^{-b_{1} / a}(\zeta)\left(p(\zeta) u^{c}(\vartheta(\zeta))\right) \mathrm{d} \zeta\right]
\end{aligned}
$$

Next, we find a lower bound for the right-hand side of (25), independent of the solution $u$. By (4) and (19), we have

$$
\begin{aligned}
u^{c}(y) & =u^{c-b_{1}}(y) u^{b_{1}}(y) \geq(d \Upsilon(y))^{c-b_{1}} u^{b_{1}}(y) \\
& \geq(d \Upsilon(y))^{c-b_{1}}\left(\Upsilon(y) w^{1 / a}(y)\right)^{b_{1}} \\
& =d^{c-b_{1}} \Upsilon^{c}(y) w^{b_{1} / a}(y), \quad \text { for } y \geq y_{2} .
\end{aligned}
$$

Since $w$ is nonincreasing, $b_{1} / a>0$, and $\vartheta(\eta)<\eta$, it follows that

$$
\begin{aligned}
u^{c}(\vartheta(\eta)) & \geq d^{c-b_{1}} \Upsilon^{c}(\vartheta(\eta)) w^{b_{1} / a}(\vartheta(\eta)) \\
& \geq d^{c-b_{1}} \Upsilon^{c}(\vartheta(\eta)) w^{b_{1} / a}(\eta) .
\end{aligned}
$$

Going back to (22), we have

$$
w^{1-b_{1} / a}\left(y_{2}\right) \geq\left(1-\frac{b_{1}}{a}\right) d^{c-b_{1}}\left[\int_{y_{2}}^{y} p(\eta) \Upsilon^{c}(\vartheta(\eta)) \mathrm{d} \eta\right] .
$$

Since $\left(1-b_{1} / a\right)>0$, by (17) the right-hand side approaches $+\infty$ as $y \longrightarrow \infty$. This contradicts (25) and completes the proof of sufficiency for eventually positive solutions.

The eventually negative solution can be dealt similarly by introducing the variables $v=-u$.

Next, we show the necessity part by a contrapositive argument. If (17) does not hold, then for each $\kappa>0$ there exists $y_{1} \geq y_{0}$ such that

$$
\int_{\eta}^{\infty} p(\zeta) \Upsilon^{c}(\vartheta(\zeta)) \mathrm{d} \zeta \leq \frac{\kappa^{(1-c / a)}}{2},
$$

for all $\eta \geq y_{1}$. We define the set of continuous functions

$$
S=\left\{u \in C([0, \infty)):\left(\frac{\kappa}{2}\right)^{1 / a} \Upsilon(y) \leq u(y) \leq \kappa^{1 / a} \Upsilon(y), \quad y \geq y_{1}\right\} .
$$

We define an operator $\Omega$ on $S$ by

$$
(\Omega u)(y)= \begin{cases}0, & \text { if } y \leq y_{1}, \\ \int_{y_{1}}^{y}\left[\frac{1}{\varsigma(\eta)}\left[\frac{\kappa}{2}+\int_{\eta}^{\infty} p(\zeta) u^{c}(\vartheta(\zeta)) \mathrm{d} \zeta\right]\right]^{1 / a} \mathrm{~d} \eta, & \text { if } y>y_{1} .\end{cases}
$$

Note that when $u$ is continuous, $\Omega u$ is also continuous on $[0, \infty)$. If $u$ is a fixed point of $\Omega$, i.e., $\Omega u=u$, then $u$ is a solution of (1).

First, we estimate $(\Omega u)(y)$ from below. By (A3), we have

$$
(\Omega u)(y) \geq \int_{y_{1}}^{y}\left[\frac{1}{\varsigma(\eta)}\left[\frac{\kappa}{2}+0\right]\right]^{1 / a} \mathrm{~d} \eta=\left(\frac{\kappa}{2}\right)^{1 / a} \Upsilon(y) .
$$

Now, we estimate $(\Omega u)(y)$ from above. For $u$ in $S$, we have $u^{c}(\vartheta(\zeta)) \leq\left(\kappa^{1 / a} \Upsilon(\vartheta(\zeta))\right)^{c}$. Then, by $(26)$,

$$
\begin{aligned}
(\Omega u)(y) & \leq \int_{y_{1}}^{y}\left[\frac{1}{\varsigma(\eta)}\left[\frac{\kappa}{2}+\int_{\eta}^{\infty} p(\zeta) u^{c}(\vartheta(\zeta)) \mathrm{d} \zeta\right]\right]^{1 / a} \mathrm{~d} \eta \\
& \leq \kappa^{1 / a} \Upsilon(y) .
\end{aligned}
$$

Therefore, $\Omega$ maps $S$ to $S$.

Next, we find a fixed point for $\Omega$ in $S$. Let us define a sequence of functions in $S$ by the recurrence relation 


$$
\begin{aligned}
v_{0}(y) & =0, \quad \text { for } y \geq y_{0}, \\
v_{1}(y) & =\left(\Omega v_{0}\right)(y)= \begin{cases}0, & \text { if } y<y_{1}, \\
\kappa^{1 / a} \Upsilon(y), & \text { if } y \geq y_{1},\end{cases} \\
v_{n+1}(y) & =\left(\Omega v_{n}\right)(y), \quad \text { for } n \geq 1, y \geq y_{1} .
\end{aligned}
$$

Note that for each fixed $y$, we have $v_{1}(y) \geq v_{0}(y)$. Using mathematical induction, we can show that $v_{n+1}(y) \geq v_{n}(y)$. Therefore, the sequence $\left\{v_{n}\right\}$ converges pointwise to a function $v$. Using the Lebesgue dominated convergence theorem, we can show that $v$ is a fixed point of $\Omega$ in $S$. This shows under assumption (26), there is a nonoscillatory solution that does not converge to zero. This completes the proof.

Theorem 2. Assume that there exists a constant $b_{2}$, the quotient of two positive odd integers such that $0<a<b_{2}<c$. If (A1)-(A4) hold and $\varsigma(y)$ is nondecreasing, then each solution of (1) is oscillatory if and only if

$$
\int_{y_{1}}^{\infty}\left[\frac{1}{\varsigma(s)} \int_{s}^{\infty} p(\zeta) \mathrm{d} \zeta\right]^{1 / a} \mathrm{~d} \eta=\infty
$$

Proof. On the contrary, we assume that $u$ is an eventually positive solution that does not converge to zero. Using the same argument as in Lemma 1 , there exists $y_{1} \geq y_{0}$ such that $u(\vartheta(y))>0$ and $\varsigma(y)\left(u^{\prime}(y)\right)^{a}$ is positive and nonincreasing. Since $\varsigma(y)>0, u(y)$ is increasing for $y \geq y_{1}$. Using $u(y) \geq u\left(y_{1}\right)$, we have

$$
u^{c}(y) \geq u^{c-b_{2}}(y) u^{b_{2}}(y) \geq u^{c-b_{2}}\left(y_{1}\right) u^{b_{2}}(y),
$$

and hence

$$
u^{c}(\vartheta(y)) \geq u^{c-b_{2}}\left(y_{1}\right) u^{b_{2}}(\vartheta(y)), \quad \text { for } y \geq y_{2} .
$$

Using (34) and $\vartheta(y) \geq \vartheta_{0}(y)$, from (13), we have

$$
\varsigma(y)\left(u^{\prime}(y)\right)^{a} \geq u^{c-b_{2}}\left(y_{1}\right) u^{b_{2}}\left(\vartheta_{0}(y)\right) \int_{y}^{\infty} p(\eta) \mathrm{d} \eta,
$$

for $y \geq y_{2}$. From $\varsigma(y)\left(u^{\prime}(y)\right)^{a}$ being nonincreasing and $\vartheta_{0}(y) \leq y$, we have

$$
\varsigma\left(\vartheta_{0}(y)\right)\left(u^{\prime}\left(\vartheta_{0}(y)\right)\right)^{a} \geq \varsigma(y)\left(u^{\prime}(y)\right)^{a} .
$$

We use this in the left-hand side of (35). Then, dividing by $\varsigma\left(\vartheta_{0}(y)\right) u^{b_{2}}\left(\vartheta_{0}(y)\right)>0$ and raising both sides to the $1 / a$ power, we have

$$
\frac{u^{\prime}\left(\vartheta_{0}((y))\right)}{u^{b_{2} / a}\left(\vartheta_{0}(y)\right)} \geq\left[\frac{u^{c-b_{2}}\left(y_{1}\right)}{\varsigma\left(\vartheta_{0}(y)\right)} \int_{y}^{\infty} p(\eta) \mathrm{d} \eta\right]^{1 / a},
$$

for $y \geq y_{2}$. Multiplying the left-hand side by $\vartheta_{0}^{\prime}(y) / \vartheta_{0} \geq 1$ and integrating from $y_{2}$ to $y$, we have

$$
\begin{aligned}
& \frac{1}{\vartheta_{0}} \int_{y_{2}}^{y} \frac{u^{\prime}\left(\vartheta_{0}(\eta)\right) \vartheta_{0}^{\prime}(\eta)}{u^{b_{2} / a}\left(\vartheta_{0}(\eta)\right)} \mathrm{d} \eta \\
& \quad \geq u^{c-b_{2}}\left(y_{1}\right) \int_{y_{2}}^{y}\left[\frac{1}{\varsigma\left(\vartheta_{0}(\eta)\right)} \int_{\eta}^{\infty} p(\zeta) \mathrm{d} \zeta\right]^{1 / a} \mathrm{~d} \eta .
\end{aligned}
$$

On the left-hand side, since $a<b_{2}$, integrating, we have

$$
\begin{aligned}
& \frac{1}{\alpha\left(1-b_{2} / a\right)}\left[z^{1-b_{2} / a}\left(\vartheta_{0}(\eta)\right)\right]_{s=y_{2}}^{y} \\
& \leq \frac{1}{\alpha\left(b_{2} / a-1\right)} z^{1-b_{2} / a}\left(\vartheta_{0}\left(y_{2}\right)\right)<\infty .
\end{aligned}
$$

On the right-hand side of (38), we use that $\varsigma\left(\vartheta_{0}(\eta)\right) \leq \varsigma(\eta)$ to conclude that (32) implies the right-hand side approaching $+\infty$, as $y \longrightarrow \infty$, which is a contradiction. Hence, the solution $u$ cannot be eventually positive.

For eventually negative solutions, we use the same change of variables as in Theorem 1 and proceed as above.

To prove the necessity part, we assume that (32) does not hold and obtain an eventually positive solution that does not converge to zero. If (32) does not hold, then for each $\kappa>0$ there exists $y_{1} \geq y_{0}$ such that

$$
\int_{y_{1}}^{\infty}\left[\frac{1}{\varsigma(\eta)} \int_{\eta}^{\infty} p(\zeta) \mathrm{d} \zeta\right]^{1 / a} \mathrm{~d} \eta \frac{\kappa^{(1-c / a)}}{2}, \quad \forall y \geq y_{1}
$$

We define the set of continuous function

$$
S=\left\{u \in C([0, \infty)): \frac{\kappa}{2} \leq u(y) \leq \kappa \text { for } y \geq y_{1}\right\} .
$$

Then, we define the operator

$$
(\Omega u)(y)= \begin{cases}0, & \text { if } y \leq y_{1}, \\ \frac{\kappa}{2}+\int_{y_{1}}^{y} \frac{1}{\varsigma(\eta)}\left[\int_{\eta}^{\infty} p(\zeta) u^{c}(\vartheta(\zeta)) \mathrm{d} \zeta\right]^{1 / a} \mathrm{~d} \eta, & \text { if } y>y_{1} .\end{cases}
$$

Note that if $u$ is continuous, $\Omega u$ is also continuous at $y=y_{1}$. Also, note that if $\Omega u=u$, then $u$ is solution of (1).

First, we estimate $(\Omega u)(y)$ from below. Let $u \in M$, we have $(\Omega u)(y) \geq \kappa / 2+0$, on $\left[y_{1}, \infty\right)$.

Now, we estimate $(\Omega u)(y)$ from above. Let $u \in M$. Then, $u \leq \kappa$ and by (40), we have

$$
\begin{aligned}
(\Omega u)(y) & \leq \frac{\kappa}{2}+\kappa^{c / a} \int_{y_{1}}^{y}\left[\frac{1}{\varsigma(\eta)} \int_{\eta}^{\infty} p(\zeta) \mathrm{d} \zeta\right]^{1 / a} \mathrm{~d} \eta \\
& \leq \frac{\kappa}{2}+\frac{\kappa}{2}=\kappa .
\end{aligned}
$$


Therefore, $\Omega$ maps $S$ to $S$. To find a fixed point for $\Omega$ in $S$, we define a sequence of functions by the recurrence relation

$$
\begin{aligned}
v_{0}(y) & =0, \quad \text { for } y \geq y_{0}, \\
v_{1}(y) & =\left(\Omega v_{0}\right)(y)=1, \quad \text { for } y \geq y_{1}, \\
v_{n+1}(y) & =\left(\Omega v_{n}\right)(y), \quad \text { for } n \geq 1, y \geq y_{1} .
\end{aligned}
$$

Note that for each fixed $y$, we have $v_{1}(y) \geq v_{0}(y)$. Using mathematical induction, we can prove that $v_{n+1}(y) \geq v_{n}(y)$. Therefore, $\left\{v_{n}\right\}$ converges pointwise to a function $v$ in $S$. Then, $v$ is a fixed point of $\Omega$ and a positive solution of (1). The proof is completed.

Example 1. Consider the differential equations

$$
\left(e^{-y}\left(u^{\prime}(y)\right)^{11 / 3}\right)^{\prime}+\frac{1}{y+1}(u(y-2))^{1 / 3}=0 .
$$

Here, $\quad a=11 / 3, \quad \varsigma(y)=e^{-y}, \quad \vartheta_{1}(y)=y-2, \quad \Upsilon(y)=$ $\int_{y_{1}}^{y} e^{11 \eta / 3} \mathrm{~d} \eta=(3 / 11)\left(e^{11 y / 3}-e^{11 y_{1} / 3}\right)$, and $c_{1}=1 / 3$. For $b \stackrel{y_{1}}{=} 7 / 3$, we have $0<c<b<a$. To check (17), we have

$$
\begin{aligned}
& \int_{0}^{\infty} p(s) Y^{c}(\vartheta(s)) \mathrm{d} s \\
& \quad=\int_{0}^{\infty} \frac{1}{\eta+1}\left(\frac{3}{11}\left(e^{11(s-2) / 3}-e^{11 y_{1} / 3}\right)\right)^{1 / 3} \mathrm{~d} s=\infty .
\end{aligned}
$$

So, every conditions of Theorem 1 hold true, and therefore, all solutions of (45) are oscillatory or converge to zero.

Example 2. Consider the differential equations

$$
\left(\left(u^{\prime}(y)\right)^{1 / 3}\right)^{\prime}+y(u(y-2))^{7 / 3}=0 .
$$

Here, $a=1 / 3, \varsigma(y)=1, \vartheta_{1}(y)=y-2$, and $c_{1}=7 / 3$. For $b=5 / 3$, we have $c>b>a$. To check (32), we have

$$
\int_{y_{0}}^{\infty}\left[\frac{1}{\varsigma(s)} \int_{s}^{\infty} p(\zeta) \mathrm{d} \zeta\right]^{1 / a} \mathrm{~d} s=\int_{2}^{\infty}\left[\int_{s}^{\infty} \zeta \mathrm{d} \zeta\right]^{3} \mathrm{~d} s=\infty
$$

So, every conditions of Theorem 2 hold true. Thus, all solutions of (47) are oscillatory or converge to zero.

\section{Conclusion}

The aim of this work is to establish necessary and sufficient conditions for the oscillation of solution to second-order half-linear differential equation. The obtained oscillation theorems complement the well-known oscillation results present in the literature. This work, as well as [31-41], leads us to pose an open problem: Can we find necessary and sufficient conditions for the oscillation of solutions to second-order differential equation

$$
\begin{array}{r}
\left.\left[r(t)\left((y(t)+p(t) y(\tau(t)))^{\prime}\right)^{\gamma}\right]^{\prime}+\sum_{i=1}^{m} q_{i}(t) y^{\alpha_{i}}\left(\tau_{i}(t)\right)\right)=0, \\
\text { for } p \in C\left(\mathbb{R}_{+}, \mathbb{R}\right) ? .
\end{array}
$$

\section{Data Availability}

No data were used to support the findings of this study.

\section{Conflicts of Interest}

The authors declare that they have no conflicts of interest.

\section{Acknowledgments}

The research was supported by the National Natural Science Foundation of China (grant nos. 11971142, 11871202, 61673169, 11701176, 11626101, and 11601485).

\section{References}

[1] H. Ahmad, T. A. Khan, P. S. Stanimirovic et al., "Modified variational iteration technique for the numerical solution of fifth order KdV type equations," Journal of Applied and Computational Mechanics, vol. 6, pp. 1220-1227, 2020.

[2] F. Mebarek-Oudina, "Convective heat transfer of titania nanofluids of different base fluids in cylindrical annulus with discrete heat source," Heat Transfer-Asian Research, vol. 48, no. 1, pp. 135-147, 2019.

[3] H. Ahmad, A. Akgül, T. A. Khan, P. S. Stanimirović, and Y.-M. Chu, "New perspective on the conventional solutions of the nonlinear time-fractional partial differential equations," Complexity, vol. 2020, p. 1, Article ID 8829017, 2020.

[4] M. Farhan, Z. Omar, F. Mebarek-Oudina et al., "Implementation of the one-step one-hybrid block method on the nonlinear equation of a circular sector oscillator," Computational Mathematics and Modeling, vol. 31, no. 1, pp. 116132,2020

[5] M. Srivastava, H. Ahmad, I. Ahmad, P. Thounthong, and N. Khan, "Numerical simulation of three-dimensional fractional-order convection-diffusion PDEs by a local meshless method," Thermal Science, p. 210, 2020.

[6] I. Ahmad, H. Ahmad, A. E. Abouelregal et al., "Numerical study of integer-order hyperbolic telegraph model arising in physical and related sciences," The European Physical Journal Plus, vol. 135, pp. 1-14, 2020.

[7] P. Thounthong, M. Khan, I. Hussain, I. Ahmad, and P. Kumam, "Symmetric radial basis function method for simulation of elliptic partial differential equations," Mathematics, vol. 6, no. 12, p. 327, 2018.

[8] H. Ahmad, T. A. Khan, P. S. Stanimirović, Y.-M. Chu, and I. Ahmad, "Modified variational iteration algorithm-II: convergence and applications to diffusion models," Complexity, vol. 2020, Article ID 8841718, 14 pages, 2020.

[9] S. Hamrelaine, F. Mebarek-Oudina, and M. R. Sari, "Analysis of MHD Jeffery Hamel flow with suction/injection by homotopy analysis method," Journal of Advanced Research in Fluid Mechanics and Thermal Sciences, vol. 58, pp. 173-186, 2019.

[10] F. Mebarek-Oudina, N. Keerthi Reddy, and M. Sankar, "Heat source location effects on buoyant convection of nanofluids in an annulus," Advances in Fluid Dynamics, pp. 923-937, 2021.

[11] I. Ahmad, M. N. Khan, M. Inc, H. Ahmad, and K. S. Nisar, "Numerical simulation of simulate an anomalous solute transport model via local meshless method," Alexandria Engineering Journal, vol. 59, no. 4, pp. 2827-2838, 2020.

[12] M. Inc, M. N. Khan, I. Ahmad, S.-W. Yao, H. Ahmad, and P. Thounthong, "Analysing time-fractional exotic options via 
efficient local meshless method," Results in Physics, vol. 19, p. $103385,2020$.

[13] A. Yokus, H. Durur, H. Ahmad, P. Thounthong, and Y.-F. Zhang, "Construction of exact traveling wave solutions of the Bogoyavlenskii equation by $\left(G^{\prime} / G, 1 / G\right)$-expansion and $\left(1 / G^{\prime}\right)$-expansion techniques," Results in Physics, vol. 19, p. $103409,2020$.

[14] S. Abdel-khalek, A. Alhag, S. M. Abo-Dahab et al., "Atomic Fisher information and entanglement forecasting for quantum system based on artificial neural network and time series model," International Journal of Quantum Chemistry, p. e26446, 2020.

[15] I. Ahmad, H. Ahmad, P. Thounthong, Y.-M. Chu, and C. Cesarano, "Solution of multi-term time-fractional PDE models arising in mathematical biology and physics by local meshless method," Symmetry, vol. 12, no. 7, p. 1195, 2020.

[16] J. Hale, Theory of Functional Differential Equationsp. 1, 2nd edition, Springer-Verlag, Berlin, Germany, 1977.

[17] S. B. Chen, S. Rashid, M. A. Noor, R. Ashraf, and Y. M. Chu, "A new approach on fractional calculus and probability density function," AIMS Mathematics, vol. 5, no. 6, 2020.

[18] F. Mebarek-Oudina, A. Aissa, B. Mahanthesh, and H. F. Öztop, "Heat transport of magnetized Newtonian nanoliquids in an annular space between porous vertical cylinders with discrete heat source," International Communications in Heat and Mass Transfer, vol. 117, p. 104737, 2020.

[19] S. Rashid, D. Baleanu, and Y. M. Chu, "Some new extensions for fractional integral operator having exponential in the kernel and their applications in physical systems," Open Physics, vol. 18, pp. 478-549, 2020.

[20] S. Rashid, H. Ahmad, A. Khalid, and Y.-M. Chu, "On discrete fractional integral inequalities for a class of functions," Complexity, vol. 2020, Article ID 8845867, 13 pages, 2020.

[21] H. Ahmad, T. A. Khan, I. Ahmad, P. S. Stanimirović, and Y.-M. Chu, "A new analyzing technique for nonlinear time fractional Cauchy reaction-diffusion model equations," Results in Physics, vol. 19, p. 103462, 2020.

[22] S.-S. Zhou, S. Rashid, S. Rashid et al., "New HermiteHadamard type inequalities for exponentially convex functions and applications," AIMS Mathematics, vol. 5, no. 6, pp. 6874-6901, 2020.

[23] O. Bazighifan, H. Ahmad, and S.-W. Yao, "New oscillation criteria for advanced differential equations of fourth order," Mathematics, vol. 8, no. 5, p. 728, 2020.

[24] H. G. Jile, S. Rashid, M. A. Noor, A. Suhail, and Y. M. Chu, "Some unified bounds for exponentially tgs-convex functions governed by conformable fractional operators," AIMS Mathematics, vol. 5, no. 6, pp. 6108-6123, 2020.

[25] B. Džurina and J. Dzurina, "Oscillation theorems for second order neutral differential equations," Computers \& Mathematics with Applications, vol. 61, no. 1, pp. 94-99, 2011.

[26] J. Džurina, "Oscillation theorems for second-order advanced neutral differential equations," Tatra Mountains Mathematical Publications, vol. 48, pp. 61-71, 2011.

[27] B. Džurina and J. Dzurina, "Oscillation theorems for secondorder nonlinear neutral differential equations," Computers \& Mathematics with Applications, vol. 62, no. 12, pp. 4472-4478, 2011.

[28] M. Bohner, S. Grace, I. Jadlovská et al., "Oscillation criteria for second-order neutral delay differential equations," Electronic Journal of Qualitative Theory of Differential Equations, vol. 60, pp. 1-12, 2017.

[29] S. R. Grace, J. Džurina, I. Jadlovska, T. Li et al., “An improved approach for studying oscillation of second-order neutral delay differential equations," Journal of Inequalities and Applications, vol. 2018, p. 11, 2018.

[30] A. Ali, "Reproducing kernel Hilbert space method based on reproducing kernel functions for investigating boundary layer flow of a Powell-Eyring non-Newtonian fluid," Journal of Taibah University for Science, vol. 13, no. 1, pp. 858-863, 2019.

[31] B. Karpuz and S. S. Santra, "Oscillation theorems for secondorder nonlinear delay differential equations of neutral type," Hacettepe Journal of Mathematics and Statistics, vol. 48, no. 3, pp. 633-643, 2019.

[32] G. E. Chatzarakis, J. Džurina, I. Jadlovská et al., "New oscillation criteria for second-order half-linear advanced differential equations," Applied Mathematics and Computation, vol. 347, pp. 404-416, 2019.

[33] J. Džurina, S. R. Grace, I. Jadlovska, T. Li et al., "Oscillation criteria for second-order Emden-Fowler delay differential equations with a sublinear neutral term," Mathematische Nachrichten, vol. 293, 2019.

[34] S. Pinelas and S. S. Santra, "Necessary and sufficient condition for oscillation of nonlinear neutral first-order differential equations with several delays," Journal of Fixed Point Theory and Applications, vol. 20, no. 27, pp. 1-13, 2018.

[35] S. Pinelas and S. S. Santra, "Necessary and sufficient conditions for oscillation of nonlinear first-order forced differential equations with several delays of neutral type," Analysis, vol. 39, no. 3, pp. 97-105, 2019.

[36] S. S. Santra, "Existence of positive solution and new oscillation criteria for nonlinear first-order neutral delay differential equations," Differential Equations \& Applications, vol. 8, no. 1, pp. 33-51, 2016.

[37] S. S. Santra, "Oscillation analysis for nonlinear neutral differential equations of second-order with several delays," Mathematica, vol. 59, no. 1-2, pp. 111-123, 2017.

[38] S. S. Santra, "Oscillation analysis for nonlinear neutral differential equations of second-order with several delays and forcing term," Mathematica, vol. 61, no. 84, pp. 63-78, 2019.

[39] S. S. Santra, "Necessary and sufficient condition for the solutions of first-order neutral differential equations to be oscillatory or tend to zero," Kyungpook Mathematical Journal, vol. 59, pp. 73-82, 2019.

[40] S. S. Santra, "Necessary and sufficient condition for oscillatory and asymptotic behaviour of second-order functional differential equations," Kragujevac Journal of Mathematics, vol. 44, no. 3, pp. 459-473, 2020.

[41] S. S. Santra, "Necessary and sufficient conditions for oscillatory and asymptotic behaviour of solutions to second-order nonlinear neutral differential equations with several delays," Tatra Mountains Mathematical Publications, vol. 75, no. 1, pp. 121-134, 2020. 